\title{
A Novel "no-touch" Robot-assisted Laparoscopic Technique Facilitates Ureteral Reconstructive Surgery
}

\author{
Kelly A. Swords, Alejandro R. Rodriguez, Mark A. Rich, Hubert S. Swana \\ Nemours Children's Clinic, Orlando, FL, University of South Florida., Tampa, FL
}

\begin{abstract}
Objectives: One of the current limitations of robotic surgery is the lack of haptic feedback. In order to overcome this drawback we developed a "No-Touch" (NT) technique utilizing the distal portion of a feeding tube to intubate and manipulate both the ureter and the renal pelvis during anastomosis.

Materials and Methods: Ureteral spatulation and pelvic reduction were performed as necessary. An 8 centimeter distal end segment of a 3 to 5 French feeding tube was used to intubate and manipulate the lumen of the proximal ureter and renal pelvis or recipient ureter during anastamosis. Manipulation of the discarded segment of the pelvis served as a handle to control and limit tissue handling. The NT technique allows for precise suture placement and prevented inadvertent backwall suture placement, thus ensuring luminal patency. No nephrostomy tubes or ureteral stents were utilized during these procedures. Patients were followed every three months for the first year with renal ultrasonography. Review of operative videos and surgical results was performed.

Results: Ten robotic pyeloplasties, one robotic pyeloureterostomy, and one robotic distal ureteroureterostomy were performed using the NT technique. No backwall suture placements, tissue crush or crimping injuries or occurred in any case. There were no intraoperative or post-operative complications. Resolution of hydronephrosis and symptoms was demonstrated in all patients after post-operative examination and ultrasonography.

Conclusions: A "No-Touch" robotic technique utilizing the distal blunt end of a feeding tube is a safe, effective, and inexpensive adjunct tool that minimizes tissue handling in robot-assisted laparoscopic surgery.
\end{abstract}

Int Braz J Urol. 2011; 37 (Video \#4): 419-420

Available at: www.brazjurol.com.br/videos/may_june_2011/Swords_419_420video.htm

\section{Correspondance address:}

Dr. Hubert S. Swana

Nemours Children's Clinic Orlando

1717 S. Orange Ave

Suite 100

Orlando, Fl 32806, USA

E-mail: hswana@nemours.org 


\section{EDITORIAL COMMENT}

Authors nicely illustrate the No Touch (NT) technique to perform anastomosis, namely robotic pyeloplasty. The use of a feeding tube facilitates manipulation of the ureter during the anastomosis. This allows for minimal tissue handling which hypothetically may lead to less tissue inju- ry and edema. Authors claim that this in turn will result in higher probability of ureteral patency. While this hypothesis is plausible, it remains to be proven. Cost efficacy and safety is premature to be determined at the present time.

Dr. Wassim Kassouf

Assistant Professor of Urology

McGill University

Montreal, Canada

wassim.kassouf@muhc.mcgill.ca 